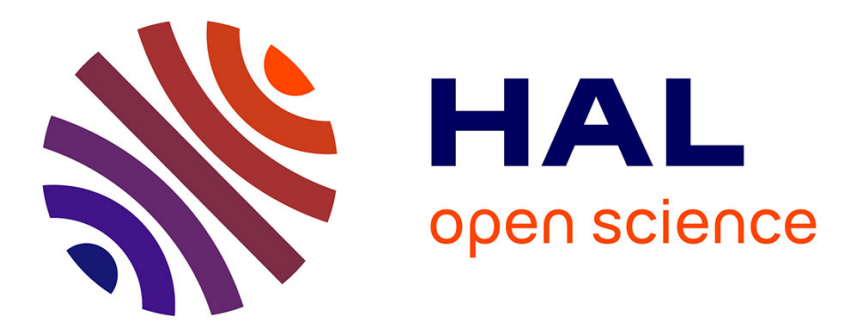

\title{
Marion Carrel, Paula Cossart, Guillaume Gourgues, Pierre-André Juven, Julien Talpin (dir.), " Vives les communes ! Des ronds-points au municipalisme ", Mouvements, $\mathrm{n}^{\circ} 101,2020$ \\ Adrien Mollaret
}

\section{To cite this version:}

Adrien Mollaret. Marion Carrel, Paula Cossart, Guillaume Gourgues, Pierre-André Juven, Julien Talpin (dir.), "Vives les communes ! Des ronds-points au municipalisme ", Mouvements, $\mathrm{n}^{\circ} 101$, 2020. Lectures, 2020, 10.4000/lectures.42367 . hal-03084310

\author{
HAL Id: hal-03084310 \\ https://hal.science/hal-03084310
}

Submitted on 21 Dec 2020

HAL is a multi-disciplinary open access archive for the deposit and dissemination of scientific research documents, whether they are published or not. The documents may come from teaching and research institutions in France or abroad, or from public or private research centers.
L'archive ouverte pluridisciplinaire HAL, est destinée au dépôt et à la diffusion de documents scientifiques de niveau recherche, publiés ou non, émanant des établissements d'enseignement et de recherche français ou étrangers, des laboratoires publics ou privés. 


\section{Marion Carrel, Paula Cossart, Guillaume Gourgues, Pierre-André Juven, Julien Talpin (dir.), "Vives les communes! Des ronds-points au municipalisme », Mouvements, $\mathrm{n}^{\circ} 101,2020$}

Adrien Mollaret

Doctorant en sociologie politique, Laboratoire PACTE, Université Grenoble Alpes.

$24 / 06 / 2020$

Pour son $101^{\mathrm{e}}$ numéro, la revue Mouvement revient sur la diversité des expériences politiques, en France et dans le monde, qui partagent un même « espoir de transformation sociale par le bas » (p. 8). Des «villes du changement» espagnoles aux Gilets jaunes, du Rojava aux listes citoyennes, ce numéro propose un «état des lieux critiques de ces expérimentations foisonnantes et hétérogènes » (p. 8), qui ont comme point commun de « repolitiser l'échelle locale [pour] en faire un espace de rupture plus ou moins radicale avec l'ordre politique et économique actuel» (p. 50). Le dossier explore trois axes pour distinguer ces pratiques diverses : l'échelle d'action, le projet politique d'ensemble et le rapport aux institutions existantes - prendre le pouvoir, l'abolir ou le subvertir. Comme à son habitude, la revue Mouvements regroupe des articles théoriques, des entretiens avec des acteurs concernés et des études de cas. Le numéro s'organise en trois parties qui abordent successivement le mouvement des Gilets jaunes, le municipalisme et le communalisme.

Le dossier s'ouvre par un article collectif relatant l'expérience de l'Assemblée des Assemblées qui, en s'inspirant des théories communalistes, a dépassé les réticences des Gilets jaunes vis-à-vis de toute forme de représentation en renouvelant les formes classiques de coordination des mouvements sociaux. Bien que cette Assemblée soit le résultat de la mobilisation d'une aile gauche militante, les auteurs montrent comment, au fur et à mesure de sa structuration, cette forme de coordination a su attirer de nouveaux Gilets jaunes moins militants et est parvenue, en partie, à représenter un mouvement politique hétéroclite. Une autre frange du mouvement a investi un répertoire d'action plus traditionnel, en rédigeant des revendications, en négociant avec le pouvoir politique ou en présentant des listes «Gilets jaunes » aux élections. Priscillia Ludosky, une figure historique du mouvement, explique comment la Convention citoyenne pour le climat a été construite en négociation 
avec des représentants des «Gilets citoyens » ${ }^{1}$. Si le mouvement des Gilets jaunes a su imposer la question de la démocratie dans l'agenda public, ses revendications n'ont été reprises que de façon partielle et euphémisée dans la séquence participative ouverte par le gouvernement. En suivant des militants de La République en marche (LREM) dans l'organisation et l'animation de réunions d'initiatives locales ${ }^{2}$, Simon Baecklandt montre que le Grand débat national de 2019, plutôt que de transformer les règles du jeu politique, a surtout joué un rôle de légitimation du pouvoir au niveau national et d'ancrage local pour le parti gouvernemental.

Les contributions de la deuxième partie, s'appuyant sur des exemples de victoires municipalistes en France, en Espagne et en Italie, posent une question centrale : «la politique change-t-elle encore les politiques » au niveau local ${ }^{3}$ ? L'article de Guillaume Gourgues rappelle que l'échelle municipale peut limiter la capacité d'action des municipalistes. Face à l'opacité et à la complexité de «l'enchevêtrement institutionnel intercommunal » (p. 52), l'auteur en appelle à une politisation des intercommunalités et à faire des métropoles le «nouveau front de lutte » (p. 53). De même, Héloise Nez montre la fragilité du mouvement municipaliste espagnol, qui repose sur le volontarisme politique de quelques élus et est largement contraint par le cadre institutionnel et administratif de la décentralisation. Les différents auteurs de cette partie se retrouvent pour dire que la clé d'un succès durable du municipalisme, c'est l'appui du changement institutionnel par une pression des citoyen-nes et des mouvements sociaux, suivant l'idée d'une «collaboration conflictuelle » (p. 104). Adrien Roux propose justement le concept de «démodynamie » pour désigner «le peuple qui manifeste» (p. 60) comme complément de la démocratie. Il

1 Collectif regroupant Gilets jaunes, chercheurs, militants écologistes et défenseurs des outils démocratiques, à l'initiative de l'association Démocratie ouverte. C'est ce collectif qui en janvier 2019 a demandé au Président de la République la création d'une assemblée citoyenne tirée au sort.

${ }^{2}$ Les RIL sont des réunions publiques regroupant une diversité d'acteurs (citoyens, élus, entreprises, syndicats...) organisées localement par des associations ou des partis politiques dans le cadre du Grand débat national.

${ }^{3}$ Arnaud Lionel, Pasquier Romain et Le Bart Christian (dir.), Idéologies et action publique territoriale. La politique change-t-elle encore les politiques?, Rennes, Presses universitaires de Rennes, coll. «Res publica », 2015. 
estime que cette forme de démocratie d'interpellation devrait être reconnue et soutenue institutionnellement au côté de la démocratie représentative, participative et directe. L'expérience des «mairies du changement » en Espagne - pourtant issues du mouvement des Indignés - montre à quel point il est difficile de trouver un équilibre entre dynamique citoyenne contestataire ascendante et dynamique institutionnelle descendante. Pour dépasser ce dilemme, entre domestication des alternatives et ensauvagement des institutions ${ }^{4}$, les municipalistes espagnols et italiens ont développé un nouveau mode de gestion administrative autour de la notion de «bien commun ». L'administration communautaire et autogérée de bien public offre des interstices réels mais fragiles entre opposition et délégation. Bendetta Celati explique qu'un des succès du municipalisme à Naples a été la création de nouvelles catégories juridiques pour donner une existence légale aux «espaces libérés ». Toutefois, la reconnaissance et la légitimation de ces pratiques ne sont pas suffisantes pour réaliser un véritable changement politique et institutionnel durable. L'autrice plaide pour inscrire la reconnaissance des biens communs dans une stratégie politique plus large. Enfin Rémi Lefebvre, dans un pertinent détour historique, montre que ces débats ne sont pas nouveaux et animaient déjà le municipalisme ouvrier, entre réformisme local et étape du processus révolutionnaire. Selon lui, l'attrait du socialisme était en grande partie dû à sa capacité à améliorer localement et concrètement la vie des ouvriers, tout en leur offrant localement des possibilités de prise de responsabilité et de politisation. Néanmoins, dans les années 1980, le municipalisme ouvrier s'est institutionnalisé, entrainant une dépolitisation de la gestion communale et une neutralisation des contre-pouvoirs. Sans toujours en avoir conscience, les «néo-municipalistes » (p. 107) d'aujourd'hui sont donc les héritiers du municipalisme ouvrier.

La troisième partie sort du cadre européen pour étudier des «utopies réelles » 5 inspirées par le communalisme. Pour Paula Cossart et Pierre Sauvêtre, il existe un vrai conflit de souveraineté entre le projet de municipalisme participatif, qui «ne désign[e] au mieux qu'un élargissement aux citoyens et aux mouvements sociaux du cercle de la

${ }^{4}$ Neveu Catherine, «Démocratie participative et mouvements sociaux: entre domestication et ensauvagement?», Participations, n ${ }^{\circ}$ 1, 2011, p. 186- 209.

\footnotetext{
${ }^{5}$ Wright Erik Olin, Utopies réelles, Paris, La Découverte, 2017.
} 
souveraineté municipale » (p. 144), et le projet communaliste, qui «doit destituer la logique de la souveraineté, des pouvoirs publics et de l'action publique, pour lui substituer celle de l'auto-gouvernement et de l'auto-organisation citoyenne » (p. 144). Au Rojava comme au Chiapas, ces expériences prennent place dans un contexte de lutte armée et de rupture avec les institutions préexistantes, l'autonomie politique se construisant «contre l'État » ou dans un «vide de pouvoir» laissé par la guerre civile. Le projet communaliste vise ici à développer des institutions démocratiques nouvelles, basées notamment sur des d'assemblées populaires et des «formes non dissociatives de délégation », pour tendre «à rendre le pouvoir d'État inutile»(p. 120). Si le Chiapas ou le Rojava sont devenus des « imaginaires mobilisateurs $»^{6}$ à travers le monde, ce ne sont pas des modèles réplicables ailleurs, tant ils sont indissociables d'un contexte culturel et politique singulier. Néanmoins, Jean-Michel Wachsberger estime que les utopies sociales n'ont pas besoin d'être légitimées par l'histoire ou de se fonder sur un «substrat communautaire »: elles peuvent aussi être créées ex-nihilo. Une des spécificités du Rojava tient à la place centrale prise par les femmes dans le processus révolutionnaire. Pour le parti des travailleurs du Kurdistan (PKK), « une libération de la société est impossible sans libération des femmes » (p. 154). Dès les années 1980, ce mouvement a encouragé les femmes à se former et à s'organiser. La fédération des femmes kurdes au Rojava (Kongreya Star), fondée en 2005, a permis la coordination et le développement du mouvement féministe kurde autour de trois piliers : un maillage territorial de Conseils et de Maisons des femmes, des académies de formation et de recherche, et des groupes d'autodéfense armée. Janet Bielh va jusqu'à dire que le nouvel agent de la révolution au $\mathrm{XXI}^{\mathrm{e}}$ siècle, c'est la femme et non le prolétaire. Plus proche de nous, le municipalisme libertaire semble connaître un regain d'activité aux États-Unis avec le renouveau des luttes anti-capitalistes au début des années 2000, et plus encore depuis l'élection de Donald Trump. Jonathan Durand-Folco et Sixtine van Outryve reviennent sur la constitution d'un mouvement qui a pour ambition de développer une alternative politique par le bas, coordonnant des assemblées locales et fédérant les mouvements sociaux, les organisations communautaires et les initiatives de démocratie radicale. Stratégiquement,

\footnotetext{
${ }^{6}$ Lebrujah Raphaël, «Le Rojava, une utopie mobilisatrice », Mouvements, 27 février 2020, en ligne :
} http://mouvements.info/rojava-utopie-mobilisatrice/. 
Jérôme Baschet appelle à la multiplication d'«espaces libérés », qui doivent être des « espaces de combat » plutôt que des « espaces refuges » (p. 127).

$\mathrm{Au}$ final, les auteurs convergent pour encourager à inscrire la question de la démocratie locale dans un projet politique plus large et ouvert sur les autres secteurs de lutte. Selon eux, l'expérimentation de l'autonomie politique locale, sous ses différentes formes, est devenue un imaginaire politique incontournable qui doit « inspirer le renouvellement de la gauche» (p. 8) dans les années à venir. On peut regretter que d'autres expériences politiques structurantes, notamment celles des zones à défendre (ZAD) et des luttes contre les grands projets inutiles, n'aient pas été intégrées dans ce dossier. En outre, la première partie sur le mouvement des Gilets jaunes aurait à notre avis mérité d'être mieux articulée avec le reste du dossier. Toutefois, ce numéro de la revue Mouvements apporte des contributions importantes nourries d'études empiriques, clarifiant les débats théoriques entre communalisme et municipalisme. Ce dossier offre donc une grille de lecture salutaire pour comprendre les potentiels, mais aussi les contraintes et les contradictions de cette diversité d'expériences participant à une «relocalisation de la politique $»^{7}$.

\footnotetext{
${ }^{7}$ Jeanpierre Laurent, «Gilets jaunes et relocalisations de la politique », Mouvements, 20 février 2020, en ligne : http://mouvements.info/relocalisation-politique-protestataire/.
} 